Williamson, D. H. \& Wilkinson, J. F. (1958). J. gen. Microbiol. 19, 198-209

\title{
The Isolation and Estimation of the Poly- $\beta$-hydroxy- butyrate Inclusions of Bacillus Species
}

\author{
By D. H. WILLIAMSON* AND J. F. WILKINSON \\ Bacteriology Department, University of Edinburgh
}

\begin{abstract}
SUMMARY : Treatment of the cells of various Bacillus spp. with an alkaline solution of sodium hypochlorite resulted in dissolution of the cells and liberation of the intracellular lipid inclusion bodies. Analyses of the isolated and purified inclusions of Bacillus cereus grown under a variety of cultural conditions showed them to contain about $89 \%$ poly- $\beta$-hydroxybutyrate and $11 \%$ ether-soluble lipid. Parallel estimations of the poly- $\beta$-hydroxybutyrate content of intact organisms by Lemoigne's chloroform extraction method showed that all of it was present in the lipid inclusions. These observations form the basis of a simple and rapid method of estimating the poly- $\beta$-hydroxybutyrate content of Bacillus spp. It consists essentially of digesting a washed bacterial suspension with a standard alkaline hypochlorite solution under standard conditions and measuring the residual turbidity.
\end{abstract}

The intracellular lipid inclusions which form such a prominent feature of the large-celled species of Bacillus have been little studied. Their avidity for oilsoluble dyes such as Sudan Black clearly indicates their content of 'fatty' substances, but little is known of the nature of these or other materials that may be present. A notable advance was made by Lemoigne, Delaporte \& Croson (1954) who were able to correlate the occurrence of lipid inclusions in Bacillus spp. with the presence in the cells of appreciable amounts of a polymer of $\beta$-hydroxybutyric acid, previously isolated from Bacillus ' $M$ ' by Lemoigne (1927). On the basis of these findings the authors concluded that the polymer was a constituent of the inclusions. Their view received some support from the observations of Weibull (1953), but has yet to be directly confirmed. The purpose of this paper is to provide such confirmation, and at the same time to describe a rapid method of estimating the poly- $\beta$-hydroxybutryate content of small samples of organisms. The method is based on the observation of Meyer (1901) that when organisms of Bacillus spp. are suspended in an alkaline solution of sodium hypochlorite, they are almost completely dissolved, only the lipid inclusions remaining intact.

\section{ME'THODS}

Organisms. The laboratory strain of Bacillus cereus (strain SC) used had the following properties: motile; Voges-Proskauer positive; catalase positive; rapid liquefaction of gelatin and coagulated serum; $\beta$-haemolytic; produced acid on glucose, sucrose, maltose, glycerol and salicin but not on lactose, duIcitol, mannitol, arabinose, rhamnose, xylose, raffinose or inulin. Abundant

* Present address: The Brewing Industry Research Foundation, Nutfield, Redhill, Surrey. 
spores and lipid inclusions were usually produced. An asporogenous mutant (strain AC) was obtained by ultraviolet irradiation of the above strain of $B$. cereus. It was recognized on an agar plate by the fact that old colonies remained fully rough instead of becoming gradually smoother as spore formation occurred. Apart from being completely asporogenous in all the culture media used, the mutant differed from the original strain in being non-motile. For optimal growth both strains required several amino acids, notably valine and leucine, but no other growth factors. The strain of Bacillus megaterium used was obtained from the National Collection of Type Cultures, Colindale, London (NCTC 7581). Aerobacter aerogenes (strain A3) was described by Duguid \& Wilkinson (1953).

Cultural methods. Unless otherwise stated, all cultures of Bacillus cereus strain AC were grown aerobically at $37^{\circ}$ for $40 \mathrm{hr}$. on a solid medium which contained (g./100 ml. distilled water): Japanese agar fibre (thoroughly washed in distilled water), 2 ; glucose, 1 ; Casamino acids (Difco), $0.005 ; \mathrm{NH}_{4} \mathrm{Cl}, 0.15$; $\mathrm{Na}_{2} \mathrm{HPO}_{4}, 0.87 ; \mathrm{NaH}_{2} \mathrm{PO}_{4}, 0.13 ; \mathrm{KCl}, 0.02 ; \mathrm{Na}_{2} \mathrm{SO}_{4}, 0.01 ; \mathrm{MgCl}_{2}, 0.002 ;$ $\mathrm{CaCl}_{2}, 0.0001 ; \mathrm{MnCl}_{2}, 0.00006 ; \mathrm{FeCl}_{3}, 0.00002$. The desired concentrations of glucose, $\mathrm{NH}_{4} \mathrm{Cl}$, phosphates, and Casamino acids were attained by adding concentrated solutions of these components, separately sterilized by light autoclaving, to the molten salts + agar base, just before pouring plates. The initial $\mathrm{pH}$ value of the medium was about $7 \cdot 3$. B. cereus (strain $\mathrm{SC}$ ) and B. megaterium were grown on nutrient agar for $24 \mathrm{hr}$. to allow sporulation to occur. Small quantities of organisms were obtained by incubating cultures in Petri dishes containing $50 \mathrm{ml}$. agar medium. The inoculum, which consisted of $0.3 \mathrm{ml}$. of a just visibly turbid suspension of organisms in $0.85 \%(\mathrm{w} / \mathrm{v})$ $\mathrm{NaCl}$, was spread evenly over the surface of the medium to give confluent uniform growth. Large samples of organisms were obtained by incubating cultures in sterile enamel trays $(40 \times 30 \times 2 \mathrm{~cm}$.). Each of these trays held c. 1.5 l. medium which was surface-inoculated with $10 \mathrm{ml}$. of a dense suspension. During incubation, these cultures were protected with loose-fitting aluminium lids and serious contamination was never encountered.

Staining methods. Lipid inclusions were stained with Sudan Black (Burdon, 1946), volutin by Albert's method as modified by Laybourne (1924), and spores by the malachite green method of Ashby (1938). Approximate estimates of cell contents of lipid and volutin inclusions were made by microscopic examination of appropriately stained smears. Several hundred organisms were examined in each case and the average number and size of the inclusions/organism recorded in terms of an arbitrary scale of ' + ' signs. In the case of the lipid inclusions the scale ranged from ' \pm ' for an average of a few small inclusions/organism through ' + ' ' ++ ' and ' +++ ' for increasing number of larger inclusions to ' ++++ ' for cultures in which nearly all the organisms were packed with masses of inclusions.

Turbidity measurements. The turbidities of suspensions of organisms and of lipid inclusions were measured with a 'Spekker' photoelectric absorptiometer, using a neutral filter. Concentrated suspensions were diluted to a point lying on the linear part of the curve relating turbidity to concentration. 
Dry-weight measurements. The dry weights of suspensions of washed organisms in distilled water were estimated by drying measured volumes to constant weight at $120^{\circ}$. Suspensions of lipid inclusions were dried to constant weight at $60^{\circ}$ in an oven, a procedure which gave the same weight as that obtained by freeze-drying.

Chemical estimations. Measurements of total nitrogen were made by a microKjeldahl procedure and of total phosphorus by the Fiske \& SubbaRow (1925) method. The ash content of isolated inclusions was determined by incinerating samples to constant weight in a platinum crucible.

Preparation of the standard alkaline hypochlorite reagent. This reagent, which was used for isolation and quantitative estimation of the lipid inclusions, was prepared as follows. Fresh bleaching powder (200 g.) was thoroughly triturated with a little distilled water and the volume made up to 11 . A litre of $30 \%(\mathrm{w} / \mathrm{v}) \mathrm{Na}_{2} \mathrm{CO}_{3}$ was added with stirring, the mixture then being left to stand for 2-3 hr. with shaking at intervals, and finally filtered through paper. The $\mathrm{pH}$ value of the greenish yellow filtrate was adjusted to 9.8 with concentrated $\mathrm{HCl}$, a flocculent precipitate which appeared during the course of this adjustment being removed by filtration after warming the solution to $37^{\circ}$. The resultant clear liquid was stored in a stoppered bottle in the refrigerator, and was stable for several months.

\section{RESULTS}

\section{Microscopical observations}

The appearance under the phase-contrast microscope of organisms of Bacillus cereus strain AC grown on the defined medium is shown in $\mathrm{Pl}$. 1, fig. 1. The identity of volutin and lipid inclusions as seen with the phase-contrast microscope was established by examining particular fields of dried smears mounted in water, the smears then being stained for volutin or lipid and the same fields re-examined. The lipid inclusions occurred predominantly at the centre of the cell and varied in size from large spherical bodies as wide as the cell, often glassy blue in colour, to small brown or black opaque bodies with welldefined outlines. Volutin granules occurred almost solely at the poles of the cells and were just visible as rather indistinct smudges. The separate identity of the two types of inclusion was strikingly demonstrated when smears were stained simultaneously for volutin and lipid. For this purpose the counterstain of Burdon's method was omitted and the smear taken instead through Albert's procedure. The lipid inclusions appeared brown against the greenstained cytoplasm, while volutin was jet black. The identification of spores under the phase-contrast microscope presented little difficulty. They were in any case absent from $B$. cereus strain AC, but in preparations of spore-bearing strains could readily be distinguished from the larger lipid inclusions by their uniform size and oval shape. Moreover, lipid inclusions were never stained by the malachite green spore stain, and spores invariably failed to take up Sudan Black.

When inclusion-bearing organisms of Bacillus cereus strain AC were suspended in the alkaline hypochlorite reagent at $37^{\circ}$, the turbidity of the sus- 
pension decreased rapidly to a low but stable value. Examination with the phase-contrast microscope of samples taken at intervals showed that this turbidimetric change was accompanied by the dissolution of the cells, the lipid and volutin inclusions being released into the medium apparently intact. The cytoplasm faded rapidly during the first few minutes of the digestion, the inclusions at the same time becoming more distinct. In a short while all that could be seen of the cells were the inclusions encased in the by now weakly visible cell membrane. At a later stage the inclusions were to be seen in violent Brownian agitation, their movements, however, being apparently restricted by the barely visible remnants of the cell wall (Pl. 1, fig. 2, is an electron micrograph at this stage of digestion). Finally, the cell wall was completely broken down and the inclusions liberated into the surrounding medium where they persisted indefinitely. At first they tended to adhere in discrete clumps, but these eventually dispersed and the preparation had the appearance shown in $\mathrm{Pl}$. 1, figs. 3, 4. Similar experiments with spore-bearing cultures of $\boldsymbol{B}$. cereus (strain SC) B. megaterium and other Bacillus spp. revealed that spores also were apparently completely dissolved.

A more direct demonstration of the lysis of spore-bearing and of spore-free organisms was provided by continuous microscopic observation of the process. For this purpose, the action of the standard reagent $(\mathrm{pH} \mathrm{9.8)}$ on organisms between slide and coverslip was rather slow, and observations were facilitated by the use of a more alkaline reagent. When a wet film of unstained organisms was made in a mixture of 2 parts of the standard reagent and one part of $10 \mathrm{~N}-\mathrm{NaOH}$, the digestion was complete in about $15 \mathrm{~min}$. at room temperature, and continuous observation of individual organisms showed the lysis to follow exactly the course described above. A particularly striking demonstration of the process was made by applying the highly alkaline reagent to organisms which had been stained by suspension in ethanolic Sudan Black. Throughout the digestion the inclusions remained stained, and their persistence and eventual release into the medium was unequivocal. It is of some interest that when these stained organisms were suspended in the standard reagent ( $\mathrm{pH} 9 \cdot 8)$, the inclusions were rapidly decolorized. This behaviour, which will be referred to again in connexion with the staining properties of the isolated inclusions, did not impair the effectiveness of the experiment; the digestion was necessarily somewhat prolonged, but it was quite possible to locate stained inclusions at the start of the experiment and follow their fate during the whole of the lytic period.

Continuous observation of several spore-bearing strains undergoing lysis revealed that spores were released into the medium at the same time as the other inclusions, but in a few minutes they became grossly swollen, lost their glassy blue refractile appearance, slowly faded away and dissolved completely.

\section{Turbidimetric observations}

In order to study the turbidimetric changes accompanying the lysis of Bacillus cereus strain AC by hypochlorite, the turbidities of saline suspensions were measured and the organisms centrifuged down and resuspended in equal 
volumes of hypochlorite reagent maintained at constant temperature. Samples were then withdrawn at intervals and their turbidities measured. The influence of $\mathrm{pH}$ value on the course of the digestion was studied by using batches of the reagent adjusted to different $\mathrm{pH}$ values with concentrated $\mathrm{HCl}$ or $\mathrm{NaOH}$, the temperature during digestion being maintained at $37^{\circ}$; the effects of temperature were assessed by using the standard reagent at $\mathrm{pH} 9 \cdot 8$. Representative results are shown in Figs. 1 and 2. In all cases the onset of lysis was rapid, but the digestion gradually slowed until a steady final value of the turbidity was reached. This steady degree of turbidity was attained more quickly at higher temperatures and at higher $\mathrm{pH}$ values, but its value was essentially independent of either of these variables, and in the examples shown had been reached by between 60 and $90 \mathrm{~min}$. Similar results were obtained with a variety of other Bacillus spp., including cultures bearing abundant spores. The relationship

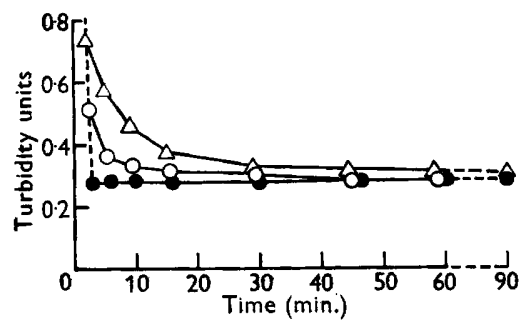

Fig. 1

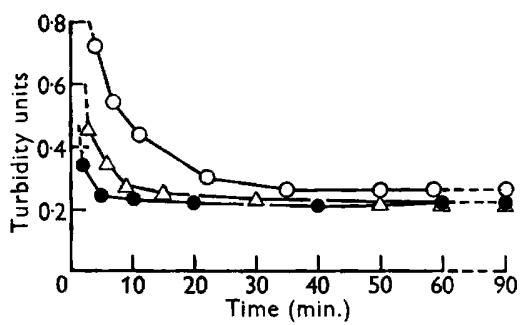

Fig. 2

Fig. 1. The effect of $\mathrm{pH}$ on the lysis of Bacillus cereus strain $\mathrm{AC}$ by standard hypochlorite at $37^{\circ}$. $\longrightarrow=$ at $\mathrm{pH} 11.6 ; \bigcirc-O=$ at $\mathrm{pH} 9.8 ; \Delta-\Delta=$ at $\mathrm{pH} 8.4$. Initial turbidity $=1.94$ units.

Fig. 2. The effect of temperature on the lysis of Bacillus cereus strain AC by standard hypochlorite at $\mathrm{pH} 9 \cdot 8$. - at $48^{\circ} ; \Delta-\Delta=$ at $37^{\circ} ; \mathrm{O}-\mathrm{O}=$ at $30^{\circ}$. Initial turbidity $=2 \cdot 40$ units.

between the initial concentration of organisms and the value of the final steady turbidity attained on treatment with hypochlorite was studied by making a series of dilutions of a suspension of inclusion-bearing organisms of $\boldsymbol{B}$. cereus strain $\mathrm{AC}$ and treating samples of these suspensions with hypochlorite ( $\mathrm{pH} \mathrm{9.8;}$ $37^{\circ}$ ) in the manner described above, the final turbidity being measured after 90 min. digestion. It will be seen from the results (Fig. 3) that the final turbidity of a suspension containing not more than the equivalent of $c .10 \mathrm{mg}$. $\mathrm{dry}-\mathrm{wt}$. organism $/ \mathrm{ml}$. was directly proportional to the initial concentration of organism. The use of more concentrated suspensions led to a final turbidity that was disproportionately high, presumably because there was insufficient hypochlorite to complete the digestion.

The above results suggested that the final turbidity attained on treatment of the organisms was due to a hypochlorite-insoluble residue, and the nature of the cytological changes occurring during lysis left little doubt that this residue was comprised essentially of the lipid and volution inclusions. It was, however, apparent from the appearance of the organisms under the phase-contrast microscope, that volutin granules were very much less refractile than the 
lipid inclusions. Aerobacter aerogenes grown under acid conditions which produce abundant volutin (Duguid, Smith \& Wilkinson, 1954) gave a turbidity equivalent to that of Bacillus cereus grown under conditions giving a minimal lipid inclusion content. Moreover, observations made during extensive studies of the growth cycle of $B$. cereus strain AC under a variety of cultural conditions showed that this strain rarely formed much volutin. It was thought, therefore, that the volutin inclusions of this strain were unlikely to contribute greatly to the final turbidity attained on treatment with hypochlorite and that this turbidity could be used to provide an estimate of the cell content of lipid inclusions. To test this hypothesis, cultures of B. cereus strain

Table 1. The relationship between the lipid inclusion content of cultures of Bacillus cereus Strain AC estimated microscopically, and the ratio of the turbidities before and after treatment with standard hypochlorite reagent.

$\begin{array}{lccccc}\begin{array}{c}\text { Microscopically estimated } \\ \text { lipid inclusion content }\end{array} & \pm & + & ++ & +++ & +++ \\ \text { Number of cultures studied } & 17 & 17 & 17 & 14 & 8 \\ \text { Mean ratio: } \frac{\text { final turbidity }}{\text { initial turbidity }} & \mathbf{0 . 0 2 4} & \mathbf{0 . 0 3 4} & 0.052 & 0.158 & 0.262 \\ \text { Standard deviation } & 0.006 & 0.006 & 0.018 & 0.024 & 0.011\end{array}$

AC containing widely different amounts of lipid inclusions (estimated by microscopical examination of stained smears) were prepared by incubation for varying periods on several versions of the basal medium, each of which had been made deficient in one of the main components, e.g. carbon, nitrogen, sulphur, potassium or phosphorus sources. Samples were treated with hypochlorite, the procedure used in this and all subsequent quantitative determinations being as follows. Washed organisms were suspended in $10 \mathrm{ml} .0 \cdot 85 \%$ $(\mathrm{w} / \mathrm{v}) \mathrm{NaCl}$ to a density equivalent to $c .1 .0 \mathrm{mg}$. dry-wt. $/ \mathrm{ml}$., a concentration lying on the linear part of the curve which relates the final turbidity attained on treatment with hypochlorite to the initial concentration of organism (Fig. 3). The suspension was centrifuged, the supernatant liquid removed and replaced with an equal volume of standard hypochlorite reagent ( $\mathrm{pH} \mathrm{9.8)}$ and the turbidity of the resulting suspension measured after $90 \mathrm{~min}$. at $37^{\circ}$. The results are shown in Table 1 , where the cultures have been grouped according to their microscopically-estimated lipid inclusion contents, and the average value for each group of the ratio of the turbidities calculated. It is clear that there was a direct statistical relationship between this ratio and the lipid inclusion content of the organisms. The scatter implicit in the standard deviations of these figures stems from the necessarily subjective nature of the microscopical estimation, rather than from inaccuracies inherent in the hypochlorite technique. This is shown by the results of the chemical analyses described below, which indicate that individual determinations with hypochlorite can be made to yield accurate estimates of the amount of lipid inclusion material.

In order to express this arbitrary measurement in more meaningful units, a 
calibration curve was constructed relating the final turbidity of the hypochlorite-treated suspension to the mass of suspended lipid inclusions. Experience showed it necessary to ensure that the suspended inclusions used to prepare this curve were dispersed to the same extent as those of actual test samples, and that the colour and refractive index of the suspending fluid was as nearly as possible the same as that of the hypochlorite reagent at the end of the digestion period. The following procedure was therefore adopted. Washed inclusion-bearing organisms of Bacillus cereus strain AC were suspended to a density equivalent to $c .8 .0 \mathrm{mg}$. dry-wt./1. in the standard hypochlorite reagent.

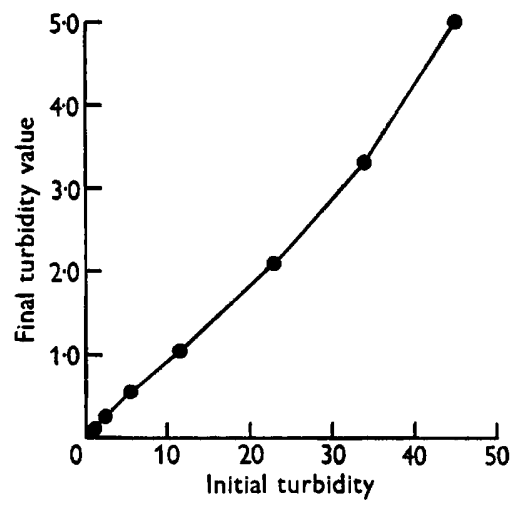

Fig. 3

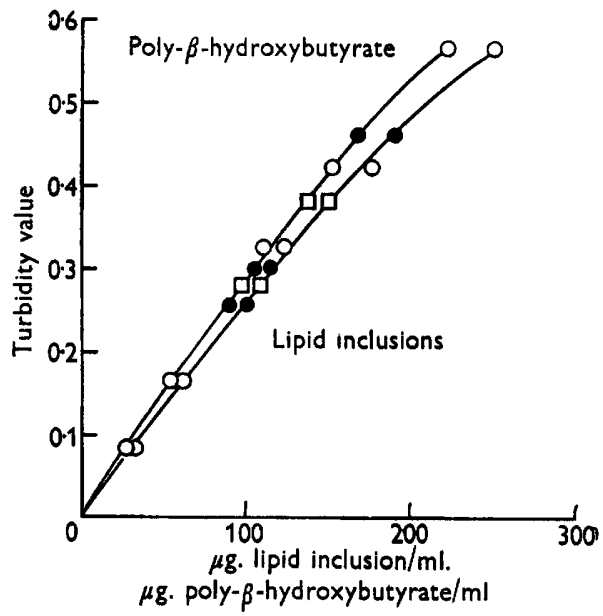

Fig. 4

Fig. 8. The relationship between the initial turbidity of a suspension of Bacillus cereus strain $A C$ and the final turbidity after treatment with standard hypochlorite under standard conditions at $\mathrm{pH} 9 \cdot 8$ and $37^{\circ}$.

Fig. 4. Calibration curve for the estimation of lipid inclusions and poly- $\beta$-hydroxybutyrate in Bacillus cereus strain AC.

at $87^{\circ}$. After stirring for $2 \mathrm{hr}$., the suspension was divided, one portion being centrifuged at high speed and the supernatant liquid passed through a fine sintered-glass filter to provide a quantity of 'used' hypochlorite. A series of dilutions of the other portion of the suspension were then made in this 'used' hypochlorite, and the turbidity of each dilution measured. Samples were centrifuged at high speed and the deposited inclusions resuspended as far as possible in distilled water and washed six times by centrifugation, a few drops of $\mathrm{Na}_{2} \mathrm{~S}_{2} \mathrm{O}_{3}$ being added to the water of the third wash in order to remove traces of free chlorine. Finally the dry weight of each sample was determined and the data plotted. Three cultures were used to prepare the calibration curve. They were grown on the standard medium for 30,40 , and $60 \mathrm{hr}$. and contained, respectively, $25 \cdot 2,16.0$ and $9 \cdot 7 \%$ of the dry weight as hypochlorite-isolated lipid inclusions. The calibration curve in Fig. 4 demonstrates that the method can be used for a wide range of inclusion content. 


\section{Chemical examination of the inclusions isolated by hypochlorite}

A washed suspension of Bacillus cereus equivalent to $c .8 \cdot 0 \mathrm{mg}$. dry-wt./ml. was centrifuged, suspended in the standard hypochlorite reagent at $37^{\circ}$ and stirred for $2 \mathrm{hr}$. Attempts to wash the resulting dense suspension of inclusions by centrifugation were unsuccessful as the inclusions were prone to clump together at the bottom of the centrifuge tube, sometimes forming tough 'buttons' which were virtually impossible to resuspend. Consequently, the suspension was diluted with an equal volume of distilled water, and dialysed against distilled water for $24 \mathrm{hr}$. Volutin granules were found to be water soluble and were removed by this treatment. On adding sufficient $\mathrm{NaOH}$ to make the suspension slightly alkaline, the inclusions could be concentrated by low-speed centrifugation and resuspended fairly easily at this stage. Traces of free chlorine were then removed by the addition of $\mathrm{Na}_{2} \mathrm{~S}_{2} \mathrm{O}_{3}$ until a negative reaction was obtained with starch-iodide reagent. Finally the dialysis was repeated and the suspension freeze-dried to form a fine white powder containing $0.10 \% \mathrm{~N} ; 0.08 \% \mathrm{P} ; 0.39 \%$ ash. Exhaustive extraction by shaking with repeated changes of dry ether at room temperature removed about $11 \%$ of the dry weight of the powder. Evaporation of the ether extract by a current of air at $60^{\circ}$ yielded a brown greasy substance. Final traces of ether were removed from it by holding in vacuo over wax chips and it was dried in vacuo over $\mathrm{P}_{2} \mathrm{O}_{5}$. The product did not show a sharp melting point; it contained $0 \cdot 14 \% \mathrm{~N}, 0.07 \% \mathrm{P}$, had a saponification index of 218 , an acid number of 60 , and gave a positive acrolein test for glycerol (Feigl, 1947). These observations suggest a mixture of triglycerides, free fatty acids, and possibly traces of phospholipids.

The fraction remaining after ether extraction of the inclusions was found to be easily and completely soluble in chloroform and appeared to be a single compound. It had a sharp melting point $\left(160^{\circ}-169^{\circ}\right)$, contained negligible traces of nitrogen and phosphorus, and had an ash content of $0 \cdot 1 \%$. Evaporation of a chloroform solution yielded the material in the form of a thin greyish translucent membrane somewhat reminiscent of plastic sheeting. Its specific gravity, determined by flotation of small pieces in sucrose solutions of known density, lay between $\mathbf{1 \cdot 2 3}$ and $\mathbf{1 \cdot 2 5}$. It was soluble in a variety of solvents, including chloroform, glacial acetic acid, pyridine, octyl alcohol, $0.5 \mathrm{~N}$-aqueous phenol, $\mathrm{N}-\mathrm{NaOH}$ and triolein and was insoluble in water, ether, acetone, ethanol, carbon tetrachloride, the alkaline hypochlorite reagent and dilute mineral acids. On heating, the material melted, charred and gave off copious white fumes with an odour characteristic of crotonic acid. The fumes condensed on the cooler parts of the tube to form needle-shaped crystals of the latter acid, melting at $c .72^{\circ}$. The material was completely saponified by boiling for several hours with $2 \cdot 5 \mathrm{~N}-\mathrm{NaOH}$, the saponification number being 640 . All these properties suggested that it was a polymer of $\beta$-hydroxybutryic acid similar to that isolated from Bacillus ' $M$ ' by Lemoigne (1927), and described by him and by Képès \& Péaud-Lenoël (1952). Confirmation of this finding was provided by an elementary analysis which gave an empirical formula of 
$\left(\mathrm{C}_{4} \mathrm{H}_{6} \mathrm{O}_{2}\right)_{n}$. The molecular weight of the polymer, determined by isothermal distillation in chloroform, was about 5000, which suggests a chain length of about 60 residues.

Examination of samples of the inclusions isolated from many different cultures of Bacillus cereus strain AC showed that the proportion of polymer in the inclusions was essentially constant (c. $89 \%$ ) and independent, over a wide range, of the inclusion content of the organisms (see, for instance, Table 2 ). It therefore seemed possible that the polymer contents of the organisms could be estimated fairly accurately as $89 \%$ of their lipid-inclusion content as

Table 2. A comparison of the hypochlorite and chloroform-extraction methods of estimating the poly- $\beta$-hydroxybutyrate contents of cultures of Bacillus cereus strain $A C$

\begin{tabular}{|c|c|c|c|c|}
\hline Culture No. & $\begin{array}{c}\text { 'Lipid inclusion' } \\
\text { content } \\
\text { by hypochlorite } \\
\text { method } \\
\text { (\% dry-wt. of } \\
\text { organisms) }\end{array}$ & $\begin{array}{c}\text { Poly- } \beta \text {-hydroxy- } \\
\text { butyrate } \\
\text { content of } \\
\text { isolated lipid } \\
\text { inclusions } \\
\text { (\% dry-wt. of } \\
\text { inclusions) }\end{array}$ & $\begin{array}{c}\text { Polymer content } \\
\text { of cells by } \\
\text { hypochlorite } \\
\text { method } \\
\text { (\% dry-wt. of } \\
\text { organisms)* }\end{array}$ & $\begin{array}{l}\text { Polymer content } \\
\text { of organisms } \\
\text { by chloroform } \\
\text { extraction } \\
\text { method } \\
\text { (\% dry-wt. of } \\
\text { organisms)* }\end{array}$ \\
\hline $\mathbf{1}$ & $42 \cdot 6$ & $89 \cdot 2$ & $38 \cdot 0$ & $36 \cdot 6$ \\
\hline 2 & 24.7 & $88 \cdot 7$ & $22 \cdot 2$ & $20 \cdot 2$ \\
\hline 3 & $19 \cdot 6$ & $89 \cdot 1$ & $17 \cdot 4$ & $17 \cdot 5$ \\
\hline 4 & $15 \cdot 4$ & $89 \cdot 1$ & - & - \\
\hline 5 & 10.7 & 89.8 & 9.5 & $9 \cdot 4$ \\
\hline & & & $1 \cdot 6$ & \\
\hline
\end{tabular}

* Calculated from data in first column on assumption that the isolated lipid inclusions contain $89 \%$ poly- $\beta$-hydroxybutyrate.

indicated by the hypochlorite technique. The correctness of this assumption was shown by experiments in which the figures obtained by following this procedure with several cultures of $B$. cereus strain AC were compared with the polymer contents of the organisms as estimated by the chloroform extraction technique of Lemoigne \& Roukhelman (1940). Large-scale cultures were used; one of them was grown on the defined medium containing $0.3 \%$ glucose, the rest were incubated for varying periods on the standard medium containing $1.0 \%$ glucose so as to yield organisms containing widely different amounts of lipid inclusions. Samples of these cultures containing the equivalent of $c$. $10 \mathrm{mg}$. dry-wt. organism were used for the hypochlorite estimations, the remainder (usually equiv. c. 5.0 g.,) being treated with chloroform. The results (Table 2) clearly show that the two results by the two methods agreed closely in the case of cultures containing between 9 and $40 \%$ of their dry weight as lipid inclusions. It thus became justifiable to calculate and construct the second curve shown in Fig. 4 . which permits the poly- $\beta$-hydroxybutyrate content of a hypochlorite-treated suspension to be determined directly from the final turbidity. The discrepancy between the two methods in the case of cells containing only traces of the polymer is not understood. However, it does not detract seriously from the value of the hypochlorite method, the chief virtues of which lie in the small size of samples required and the rapidity with which accurate results can be obtained. 


\section{Staining properties of the isolated inclusions}

The most significant result was obtained with the Sudan dyes. Both Sudan Black and Sudan III stained the inclusions in situ, the former an intense blueblack, the latter a pale red. Both these stains, however, failed to colour the isolated inclusions. This was not due to dissolution of a sudanophilic component in the unprotected inclusions by the solvent $(70 \%, \mathrm{v} / \mathrm{v}$ ethanol in water) used to carry the dyestuffs, since the isolated inclusions could not be stained even when mounted in saturated solutions of Sudan Black in either ethylene glycol or $50 \%$ diacetin, both solvents which are almost incapable of dissolving lipids. Moreover, the lack of sudanophilia of the isolated inclusions was in agreement with the previously noted observation of the decolorization of Sudan Black-stained inclusions by the standard alkaline hypochlorite reagent. Thus it was clear that the isolated inclusions were no longer sudanophilic. No staining occurred with Albert's stain, Ashby's spore stain, the mercuric/bromphenol blue test for protein (Mazia, Brewer \& Alfert, 1953) or the Feulgen reaction.

\section{DISCUSSION}

The hypochlorite method of estimating poly- $\beta$-hydroxybutyrate is rapid, simple to perform, and requires only the equivalent of $c 10 \mathrm{mg}$. dry-wt. of organisms. It is thus particularly suitable for experiments involving frequent examination of a limited amount of culture material, and in the case of cultures containing moderate or large amounts of the polymer gives results which agree closely with those obtained by the much more cumbersome method of chloroform extraction. The discrepancy between the results given by the two methods in the case of organisms containing only traces of the polymer does not impose a serious limit on the practical utility of the method, since the 'largecelled' species of Bacillus usually contain considerable quantities of poly- $\beta$ hydroxybutyrate.

Although the asporogenous Bacillus cereus strain AC was the main subject of the present study there is no reason why the method should not be applicable to other members of the genus. A necessary preliminary to any such application is the construction of a calibration curve which relates the polymer content of cultures to the turbidities attained on treating the same cultures with hypochlorite. The calibration curve described in the present study was constructed by first determining the relationship between the turbidity and dry mass of the suspended inclusions, and then showing that the isolated inclusions contained approximately $89 \%$ poly- $\beta$-hydroxybutyrate. Alternatively calibration can be achieved directly by relating the final turbidities attained on treating known concentrations of organisms with hypochlorite, to the amount of poly- $\beta$-hydroxybutyrate in the organisms, as determined by chloroform extraction. Thus Macrae \& Wilkinson (1958) prepared a calibration curve for the polymer content of Bacillus megaterium strain KM. It should be noted, however, that the method cannot be validly applied to a particular strain unless it is first shown that the shape and position of the 
calibration curve is independent of the cell content of polymer. This is most easily done by ensuring that several cultures containing widely divergent amounts of lipid inclusions are used for the preparation of the calibration curve. Some indication of the reproducibility of the method is given by the fact that, as the result of experience, duplicate determinations were considered unnecessary. A large number of tests made in duplicate showed that the difference between a pair of determinations was never more, and usually less, than $3 \%$ of their mean.

The poly- $\beta$-hydroxybutyrate content of the lipid inclusions of Bacillus spp. was first deduced by Lemoigne et al. (1944). Their evidence was indirect, being based on finding the polymer only in species bearing lipid inclusions. The present demonstration of the polymer in the isolated and apparently morphologically intact lipid inclusions of $B$. cereus provides a direct confirmation of their suggestion. Unfortunately, the method of isolation is such that little can be deduced about other possible constituents of the inclusions in situ. Any polysaccharides, proteins, etc., which might be present would be unlikely to avoid destruction or dissolution by the reagent unless they happened to be inaccessible to it, and the lack of these materials in the isolated inclusions does not rule out the possibility of their existence in the inclusions in situ. That some degradation of the inclusions does occur during isolation is shown by their loss of sudanophilia. It may be assumed that the inclusions in situ contain a sudanophilic component as an entity distinct from the poly- $\beta$-hydroxybutyrate. The existence of such a component is shown by the finding in this laboratory that small pieces of the polymer, extracted with either chloroform or hypochlorite, could not be stained with Sudan dyes, an observation that agrees with the statement of Cain (1950) concerning the non-sudanophilic nature of solid lipids. Thus the sudanophilia of the inclusions in situ is almost certainly not due to the presence in them of the polymer, but must be attributed to a sudanophilic component which is either removed by hypochlorite treatment or in some other way rendered non-sudanophilic. It is possible that the ether-soluble component of the isolated inclusions is an altered form of this sudanophilic material, but unfortunately it cannot even be assumed with certainty that the ether-soluble lipid is in fact a true constituent of the inclusions. It is conceivable that it merely represents cytoplasmic lipid which adheres to the inclusions during the isolation process. The answers to these problems are to be found in analysing lipid inclusions isolated by other and less drastic techniques.

The authors are grateful to Dr C. 'T. Greenwood for a molecular weight determination, to Dr J. W. Minnis for an elementary analysis, and to Dr J. P. Duguid for many helpful suggestions. One of us (D.H.W.) is indebted to the University of Edinburgh for the award of Post-graduate Studentships for the years 1953-55, and to the Agricultural Research Council for the award of a Research Studentship for 1955-56. 

Journal of General Microbiology, Vol. 19, No. 1

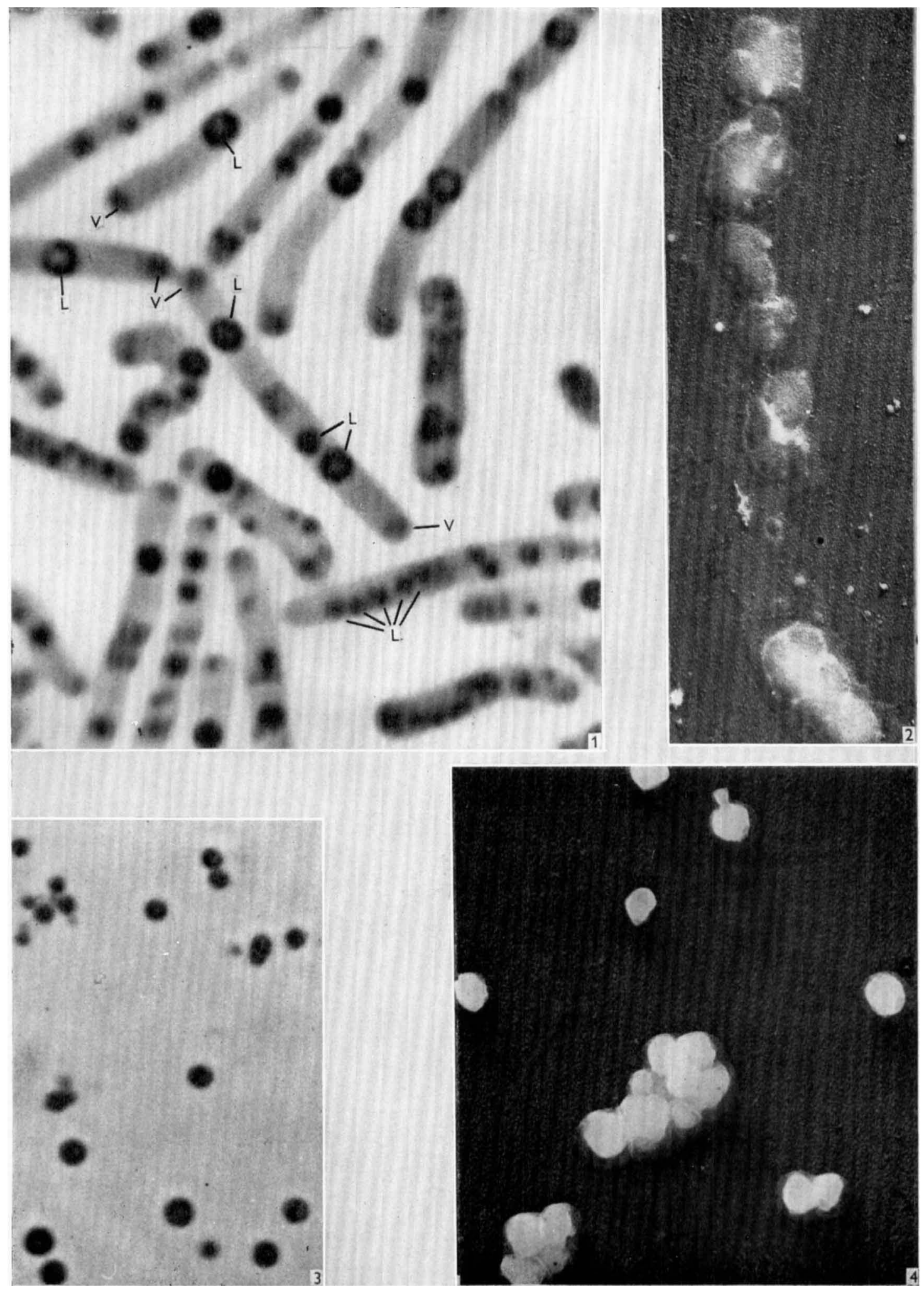

D. H. Williamson \& J. F. Wilkinson-Estimation of POLY- $\beta$-hydroxybutyrate.

Plate 1 


\section{REFERENCES}

Ashby, G. K. (1938). Simplified Schaeffer spore strain. Science, 87, 443.

Burdon, K. L. (1946). Fatty material in bacteria and fungi revealed by staining dried, fixed slide preparations. J. Bact. 52, 665 .

CaIn, A. J. (1950). The histochemistry of lipoids of animals. Biol. Rev. 25, 73.

Duguid, J. P., Smith, I. W. \& Wilkinson, J. F. (1954). Volutin production in Bacterium aerogenes due to development of an acid reaction. J. Path. Bact. 67, 289.

Duguid, J. P. \& Wilkinson, J. F. (1953). The influence of cultural conditions on polysaccharide production by Aerobacter aerogenes. J. gen. Microbiol. 9, 174.

FeIGL, F. (1947). The Quantitative Analysis by Spot Tests, 3rd edition, p. 408. New York: Elsevier Publishing Co. Inc.

Fiske, A. \& SubbaRow, Y (1925). The colorimetric determination of phosphorus. J. biol. Chem. 66, 375.

Képk̀s, A. \& PéAud-LenoËL, C. (1952). Sur les propriétés et la constitution des lipides $\beta$-hydroxybutyriques. C.R. Acad. Sci., Paris, 234, 756.

LAybourne, R. L. (1924). A modification of Albert's stain for the diphtheria bacillus. J. Amer. med. Ass. 83, 121.

Lemoigne, M. (1927). Études sur l'autolyse microbienne. Origine de l'acide $\beta$-oxybutyrique formé par autolyse. Ann. Inst. Pasteur, 41, 148.

Lemoigne, M., Delaporte, B. \& Croson, M. (1944). Contribution a l'étude botanique et biochimique des bactéries du genre Bacillus. 2. Valeur du test des lipides $\beta$-hydroxybutyriques pour la characterisation des espèces. Ann. Inst. Pasteur, 70, 224.

Lemoigne, M. \& Roukhelman, N. (1940). Caractérisation et évolution des produits de déshydration et de polymérisation de l'acide $\beta$-hydroxybutyrique, Ann. Ferment. 5, 527.

Macrae, R. M. \& Wilkinson, J. F. (1958). Poly- $\beta$-hydroxybutyrate metabolism in washed suspensions of Bacillus cereus and Bacillus megaterium. J. gen. Microbiol. 19, 210.

Mazia, D., Brewer, P. A. \& Alfert, M. (1953). The cytological staining and measurement of protein with mercuric bromophenol blue. Biol. Bull., Wood's Hole, 104-105, 57.

Meyer, A. (1901). Notiz über das Verhalten der Sporen und Fettropfen der Bakterien gegen Eau de Javelle und gegen Chloralhydratlösung. Zbl. Bakt. (1 Abt. Orig.) 29, 809.

WeIBUld, C. (1953). Characterisation of the protoplasmic constituents of Bacillus megaterium. J. Bact. 66, 696.

\section{EXPLANATION OF PLATE}

Cultures of Bacillus spp. grown for $40 \mathrm{hr}$. at $30^{\circ}$ on the standard defined medium.

Fig. 1. Bacillus cereus strain AC. Phase contrast of wet film. $\times 600$. Note lipid inclusions (L) and volutin inclusions (V).

Fig. 2. Bacillus megaterium incompletely digested with hypochlorite. The lipid inclusions are still encased in a remnant of cell material. Electron micrograph of formalin-fixed organism, shadowed at $15^{\circ}$ with gold + palladium. $\times 13,000$.

Fig. 3. Lipid inclusions of Bacillus cereus strain AC, isolated after hypochlorite treatment. Phase contrast of wet film. $\times 5,000$.

Fig. 4. Lipid inclusions of Bacillus cereus strain AC isolated after hypochlorite treatment. Electron micrograph after osmic acid fixation, shadowed at $15^{\circ}$ with gold + palladium. $\times 15,000$.

(Received 4 March 1958) 